\title{
Tracheal agenesis: optimization of computed tomography diagnosis by airway ventilation
}

\author{
Marirosa Cristallo Lacalamita $^{1,6} \cdot$ Sebastien Fau ${ }^{2} \cdot$ Aurelie Bornand $^{3} \cdot$ Isabelle Vidal $^{4}$. \\ Antonella Martino $^{5}$ • Isabelle Eperon ${ }^{5} \cdot$ Seema Toso $^{1}$ • Anne-Laure Rougemont ${ }^{3}$. \\ Sylviane Hanquinet ${ }^{1}$
}

Received: 19 June 2017 /Revised: 29 September 2017 / Accepted: 1 November 2017 / Published online: 17 November 2017

(C) Springer-Verlag GmbH Germany, part of Springer Nature 2017

\begin{abstract}
Tracheal agenesis is a rare and often lethal congenital defect that leads to airway emergency at birth. Computed tomography (CT) is the modality of choice to evaluate anomalous tracheal anatomy. The absence of spontaneous aeration of the tracheobronchial tree in children with tracheal agenesis makes CT interpretation difficult. We describe a procedure of airway management applied in two newborns with suspected tracheal agenesis. Correct airway management was performed immediately prior to CT examination by airway ventilation, with bag-valve mask alone in one case, and attached to an endotracheal tube placed into the esophagus in the other case. The images allowed for classification of tracheal agenesis. Computed tomography with appropriate airway ventilation is fundamental for the diagnosis of tracheal agenesis.
\end{abstract}

Keywords Airway ventilation - Computed tomography · Newborn · Tracheal agenesis · Tracheoesophageal fistula

Marirosa Cristallo Lacalamita

Marirosa.CristalloLacalamita@hcuge.ch

1 Department of Pediatric Radiology, University Hospital of Geneva, Geneva, Switzerland

2 Division of Neonatology and Pediatric Intensive Care, University Hospital of Geneva, Geneva, Switzerland

3 Division of Clinical Pathology, University Hospital of Geneva, Geneva, Switzerland

4 Department of Pediatric Surgery, University Hospital of Geneva, Geneva, Switzerland

5 Department of Gynaecology and Obstetrics, Division of Obstetrics, University Hospital of Geneva, Geneva, Switzerland

6 Present address: Unité de Radiopédiatrie, Hôpital Universitaire des Enfants, 6 rue Willy Donzé, 1211 Genève 14, Geneva, Switzerland

\section{Introduction}

Tracheal agenesis is a rare congenital defect resulting in complete or partial absence of the trachea below the larynx with a prevalence at birth of 1 in 50,000, with a male predominance and mortality of $85 \%$ [1].

The simple and often-used Floyd classification consists of three types, based on the presence and length of the tracheal remnant (Fig. 1) [2]. Little is known about the pathogenesis of tracheal agenesis but most theories are based on failure of development of the tracheoesophageal septum, which divides the foregut into a ventral portion, the laryngotracheal tube, and a dorsal portion, the esophagus [3]. CT examination is the procedure of choice for clearly delineating the anomalous tracheal anatomy and showing associated malformations [4]. The cases reported in the literature lack some important considerations about technical details of CT procedure for optimizing the diagnosis of tracheal agenesis $[4,5]$. In newborns with tracheal agenesis, airways cannot be spontaneously aerated, consequently they are difficult to analyze at CT. We report two newborns with tracheal agenesis confirmed by emergency CT, in order to demonstrate that proper airway management just before $\mathrm{CT}$ is essential for diagnosis and correct classification of tracheal agenesis.

\section{Description}

\section{Patients}

Patient 1 was a 35 -week-gestation boy (birth weight $2600 \mathrm{~g}$ ) with severe respiratory distress immediately after delivery (Apgar score: 1/1/5). Fetal follow-up and delivery had been performed at an outside institution. The pregnancy had been 


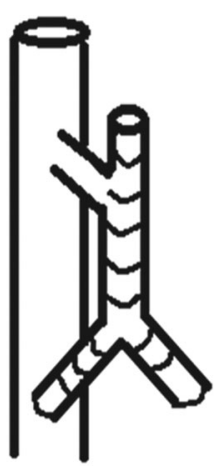

Type I

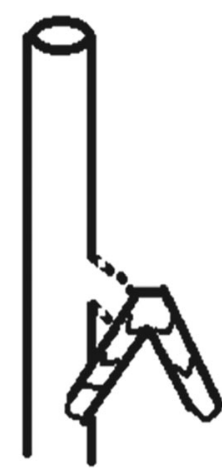

Type II

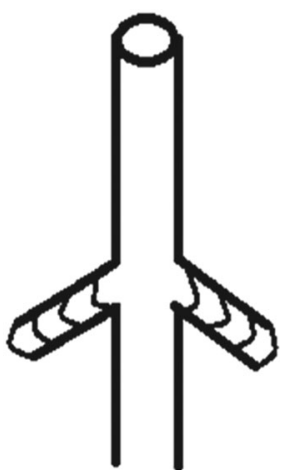

Type III
Fig. 1 Schematic representation of the classification of tracheal agenesis as described by Floyd et al. [2]. Type I: agenesis of the proximal trachea, with a distal segment of the trachea and normal carina connected with the esophagus by a tracheo-esophageal fistula. Type II: agenesis of the entire trachea, normal carina and main bronchi with or without tracheoesophageal fistula. Type III: trachea and carina are absent and the main bronchi arise separate from the esophagus

complicated by polyhydramnios during the third trimester. After delivery the baby was supported with ventilation by bag-valve mask. Four intubation attempts by endotracheal tubes of different sizes and lengths failed. The newborn was urgently transferred to our neonatal intensive care unit to be evaluated with flexible laryngoscopy, where no tracheal opening was visible and a tracheoesophageal fistula was suspected. An emergency chest CT scan was requested to clarify the tracheal anatomy. Because of the long-segment tracheal defect, a multidisciplinary discussion concluded that the anomaly could not be corrected. Life support was discontinued in agreement with the family, and the infant died. Autopsy was refused.

Patient 2 was a 36-week-gestation girl (birth weight $2290 \mathrm{~g}$ ) delivered by Cesarean section for polyhydramnios diagnosed at 32-week ultrasound. At delivery the child developed severe respiratory distress despite breathing movements, with poor adaptation (Apgar score: 1/1/3). Because several attempts to pass an endotracheal tube into the larynx beyond the vocal folds failed, tracheal agenesis was suspected and the tube was finally set into the esophagus. Laryngoscopy in the delivery room confirmed the tracheal agenesis. Because of insufficient oxygenation the neonatology team decided to put the baby on veno-arterial extracorporeal membrane oxygenation. The baby was then safely transported for a CT scan. Because of the poor prognosis for surgical correction of long tracheal defects, the parents agreed to stop life support. The child died. Autopsy confirmed the tracheal agenesis, with a blind-ending larynx $0.5 \mathrm{~cm}$ below the vocal cords, and the presence of a fistula between the right main bronchus and the esophagus (Fig. 2).

\section{Computed tomography technique}

A single-source 64-slice CT scanner (Edge; Siemens, Erlangen, Germany) was used with the following parameters: 0.6-mm collimation, 2-mm slice thickness, 1-mm reconstruction interval, $70 \mathrm{kV}$ and $90 \mathrm{mAs}$. In both cases ventilation maneuvers were manually performed by neonatologists using a bag-valve-mask equipped with a manometer, not connected to a positive-pressure airway system.

In patient 1 , a chest $\mathrm{CT}$ scan without contrast medium was preceded by several ventilation maneuvers by a bag-valve mask without endotracheal tube, suspended during the CT scan. In patient 2 , a thoraco-abdominal $\mathrm{CT}$ scan was performed after previous ventilation maneuvers by a bag-valve mask attached to an endotracheal tube (diameter: $2.5 \mathrm{~mm}$, depth at lip: $5.5 \mathrm{~cm}$ ). Numerous previous attempts of peripheral intravenous cannula insertion were unsuccessful. The acquisition delay was $20 \mathrm{~s}$ after manual injection of contrast medium (5 ml Accupaque 300; GE Healthcare) into the aorta by an umbilical artery catheter (to bypass filtering by extracorporeal membrane oxygenation and to evaluate mediastinal arterial anatomy), followed by a 35-s delayed acquisition. Because ventilation pressures were initially low, airways were not visible on the first CT. Thus the endotracheal tube was advanced into the esophagus at a distance of $11 \mathrm{~cm}$ from the lips. Ventilation pressures were gradually increased to peak pressure of $45 \mathrm{~cm} \mathrm{H}_{2} \mathrm{O}$ and expiratory pressure of $20 \mathrm{~cm}$ $\mathrm{H}_{2} \mathrm{O}$ to obtain good thoracic expansion and to improve airway distension. A second $\mathrm{CT}$ acquisition was repeated 5 min later. Multiplanar reconstructions, minimum-intensity projection and volume-rendered reconstructions were retrospectively performed using the OsiriX software.

\section{Computed tomography findings}

In patient 1 , a tracheal lumen was not identified. The two main bronchi connected by a normal carina were evident and a tracheoesophageal fistula was suspected between the right main bronchus and the esophagus, compatible with type II tracheal agenesis. A distance of $36 \mathrm{~mm}$ between the blindending larynx and the carina was measured. Minimumintensity projection reformatted and volume-rendered reconstructions better displayed the anomalies (Fig. 3).

In patient 2, on the first CT scan the tracheal lumen was not seen in the superior mediastinum. The carina and main bronchi were not visible, and both lungs were completely collapsed. An enhancing nodule of the gastric wall was discovered, which at autopsy was identified as an ectopic pancreas. A partial unilateral sacral agenesis suspected on babygram was confirmed (Fig. 4). On the chest CT repeated after advancing the endotracheal tube into the esophagus, a normal carina connecting the two main bronchi was seen and the trachea was absent below the larynx, compatible with a type 
Fig. 2 Autopsy of patient 2. a, b Hematoxylin and eosin wholemount views. The sagittal plane (a) demonstrates the blind-ending larynx $(L)$ and the transverse section $(b)$ shows the thyroid gland $(T g)$ in direct contact with the esophagus $(E)$. The trachea is totally absent. c Macroscopic findings: the presence of a fistula is confirmed (metal rod) between the bronchus $(B)$ and the esophagus $(E)$

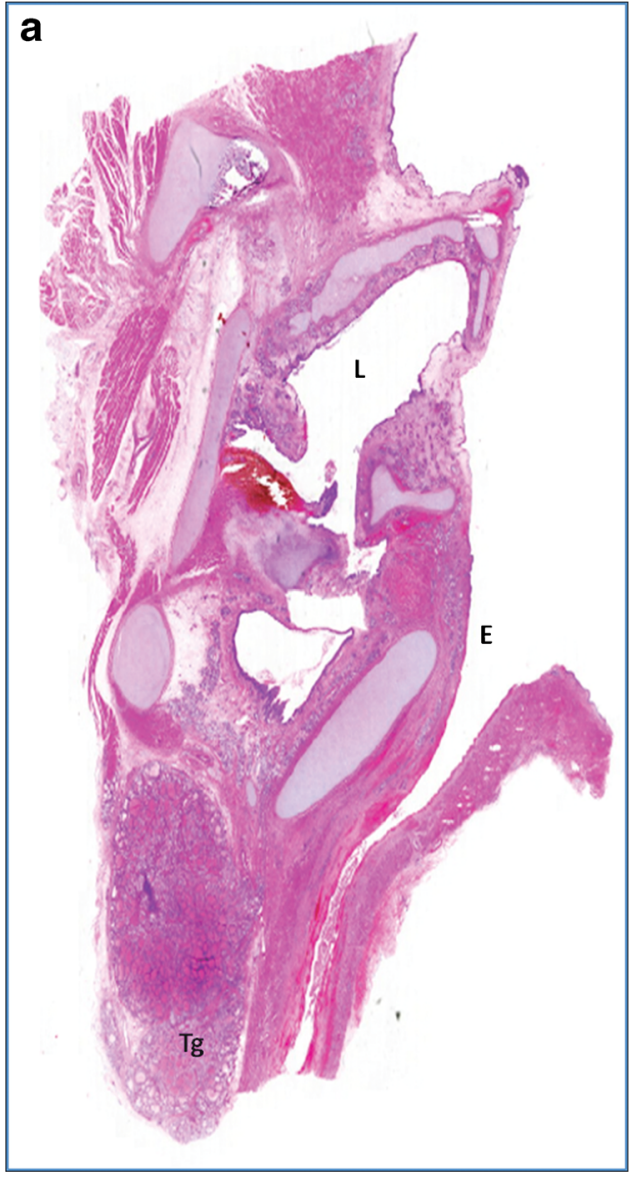

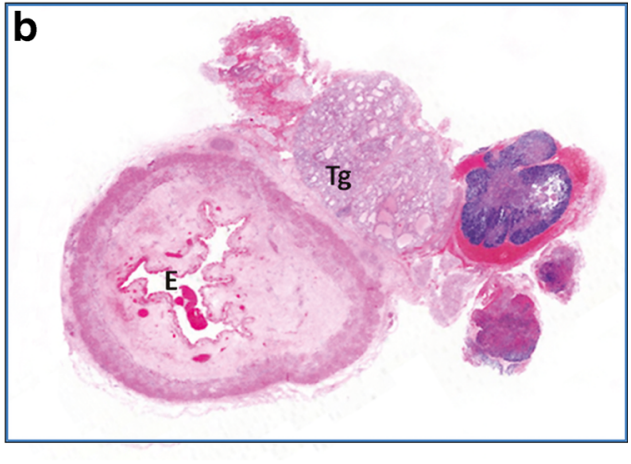

c

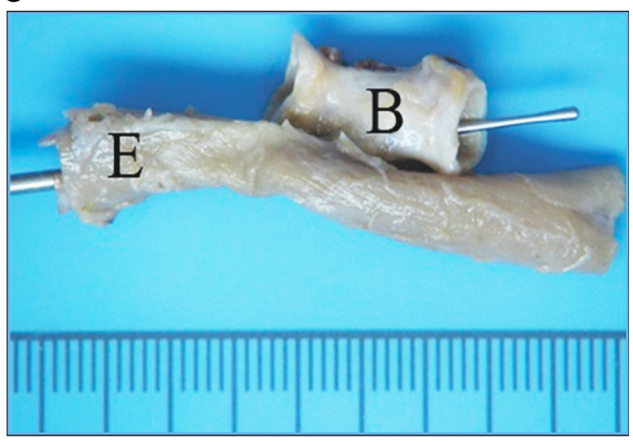

II tracheal agenesis. A tracheoesophageal fistula was not recognized but was suspected because airways were visible only after the adequate esophageal ventilation. Bronchial development and lung lobes appeared normal (Fig. 5).

\section{Discussion}

Tracheal agenesis is a rare anomaly that is part of a multiplemalformation syndrome in $>90 \%$ of cases, but it can also be isolated. The most common anomalies reported in conjunction with tracheal agenesis are the TARCD (tracheal agenesis, radial ray anomalies, congenital cardiac defects and duodenal atresia) or VACTERL associations (vertebral anomalies, anal atresia, cardiac abnormalities, tracheoesophageal fistula and/ or esophageal atresia, renal and limb malformations) [4, 5]. Annular pancreas can be also associated with a tracheal agenesis [6]. In our two cases of tracheal agenesis, one was associated with an imperforate anus and the other with both pancreatic heterotopia and partial unilateral sacral agenesis.

The prenatal diagnosis of tracheal agenesis is very difficult. In the literature seven cases are reported, detected either by antenatal ultrasound or fetal MRI [4, 7]. Antenatal ultrasound findings of tracheal agenesis depend on the presence or absence of a tracheoesophageal fistula. If tracheal agenesis is isolated without other fetal anomalies, there are no other signs to alert the sonographer apart from polyhydramnios, reported in up to $50-72 \%$ of cases.

Postnatal diagnosis can be suspected in an emergency setting by a combination of clinical signs: neonatal respiratory distress, no audible cry, failure of endotracheal intubation, and Apgar score below 7 at $5 \mathrm{~min}$ [4]. Multidisciplinary management is necessary, with effective and rapid communication among the teams of neonatology, anesthesia, radiology and surgery. While respiratory management can be used to assist in temporary prolongation of life, infants with a complete absence of the trachea tend to die within hours of birth because a permanent airway cannot be created. Currently, this anomaly is incompatible with life. In the majority of cases reported in the literature diagnosis was confirmed operatively or at autopsy; nevertheless CT is the most practical means for rapid and noninvasive diagnosis [4].

Clinical concerns include maintaining a patent airway, oxygen saturation, ventilation strategies and hemodynamic stability as far as possible prior to transferring to the CT room. Proper airway stabilization and ventilation improve CT imaging so that fewer additional maneuvers are necessary once in CT. Nevertheless, it is important to remember that ventilation 

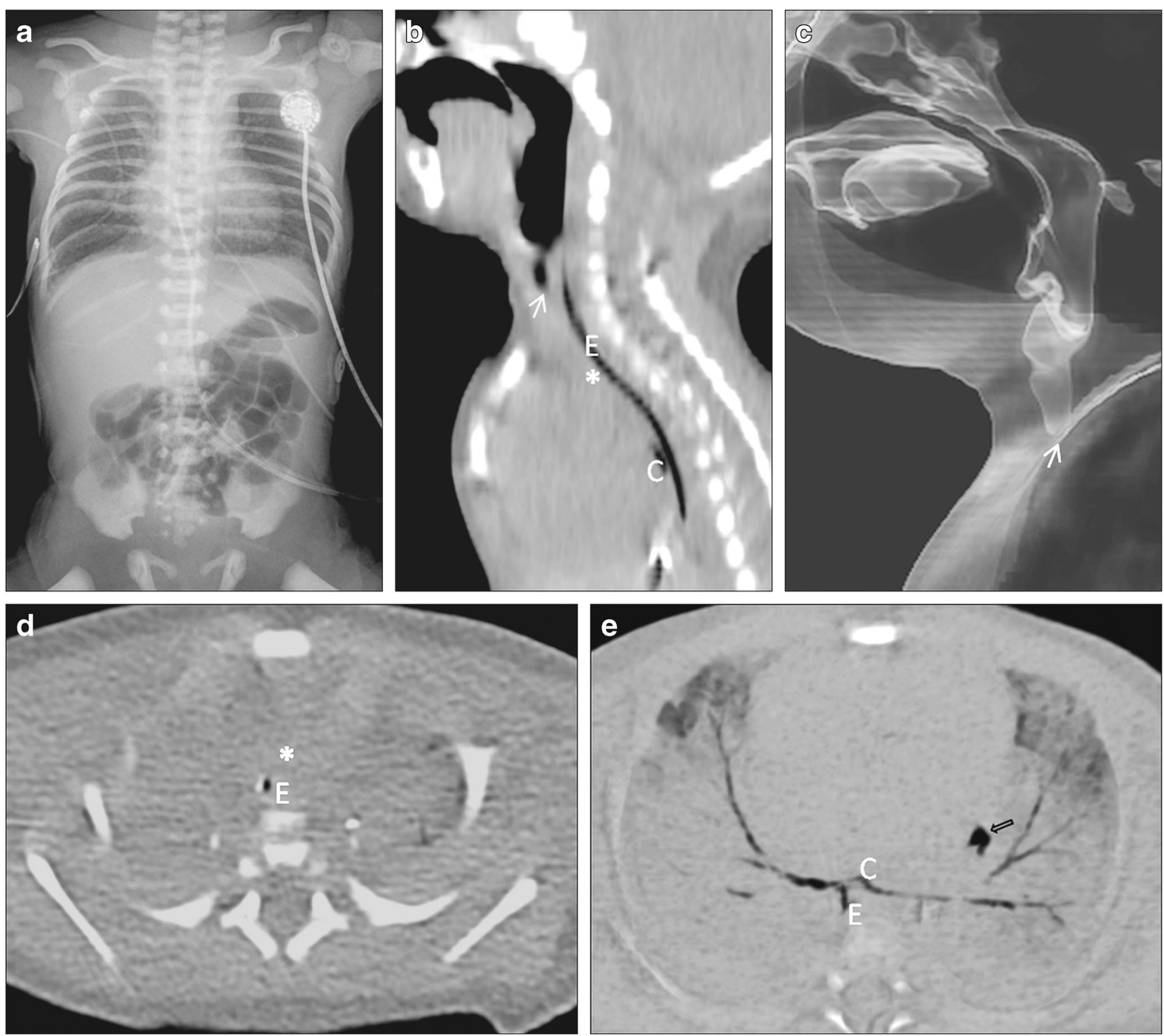

Fig. 3 Imaging of patient 1. a Anteroposterior radiograph shows lung hyperinflation from ventilation by bag-valve mask during the exams and the presence of a nasogastric tube and an umbilical venous catheter. b, c Sagittal reformat $(b)$ and volumetric reconstructions $(c)$ of CT scan show the larynx terminating in a blind-ended sac (arrow). No tracheal lumen (asterisk) is recognized in the superior mediastinum. Esophagus $(E)$,

and attempted resuscitation take priority over diagnostic procedures.

In newborn babies with tracheal agenesis, the only possible ventilator trajectory from pharynx to trachea is via the esophagus and the tracheoesophageal fistula. The thinner and longer the esophagotracheal fistula, the more limited the passage of air. Resuscitation with powerful bag ventilation might permit enough lung ventilation to maintain minimum oxygenation. This ventilation can be given by bag-valve mask or by an endotracheal tube inserted in the esophagus. When available on site, a more efficient way to maintain blood oxygenation is carina $(C)$. d, e Axial CT images: the two main bronchi arise from a normal carina $(C)$ and a suspected tracheo-esophageal fistula between the right main bronchus and esophagus $(E)$, as in type II tracheal agenesis. The lungs are atelectatic, and pneumomediastinum is present (open arrow in $\mathbf{e}$ )

to put the patient on extracorporeal membrane oxygenation, either veno-venous either veno-arterial.

The CT diagnosis of tracheal agenesis is made in the absence of a tracheal air column. On the other hand, CT can also be used to measure the extent of the length of the defect with the multiplanar reconstruction images and evaluate the anatomical relationship of the tracheal malformation.

The cases reported in the literature lack some important considerations about technical details of CT for optimizing the diagnosis of tracheal agenesis $[4,5]$. First, it is important to underline the need for ventilation maneuvers prior to the 

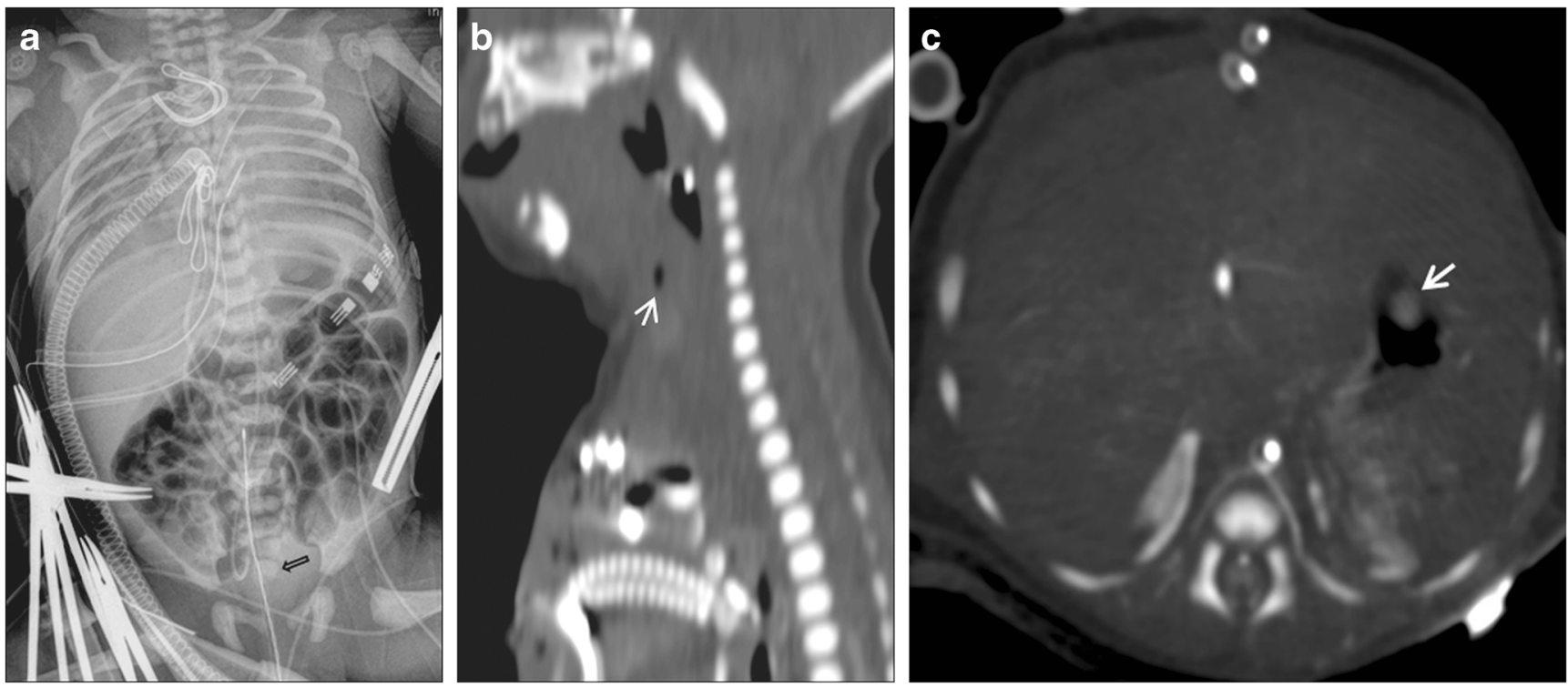

Fig. 4 Imaging of patient 2. a Anteroposterior babygram demonstrates poorly aerated lungs and gaseous distension of small-bowel loops. Several tubes are present from the extracorporeal membrane oxygenation support, umbilical artery and vein catheters, and rectal probe. There is partial unilateral sacral agenesis (arrow). b, c Sagittal reconstruction $(b)$ and axial image (c) from initial CT scan demonstrate a laryngeal blind-ended sac and tracheal agenesis (arrow in b) and reveal an enhancing nodule in the anterior gastric wall (arrow in c)
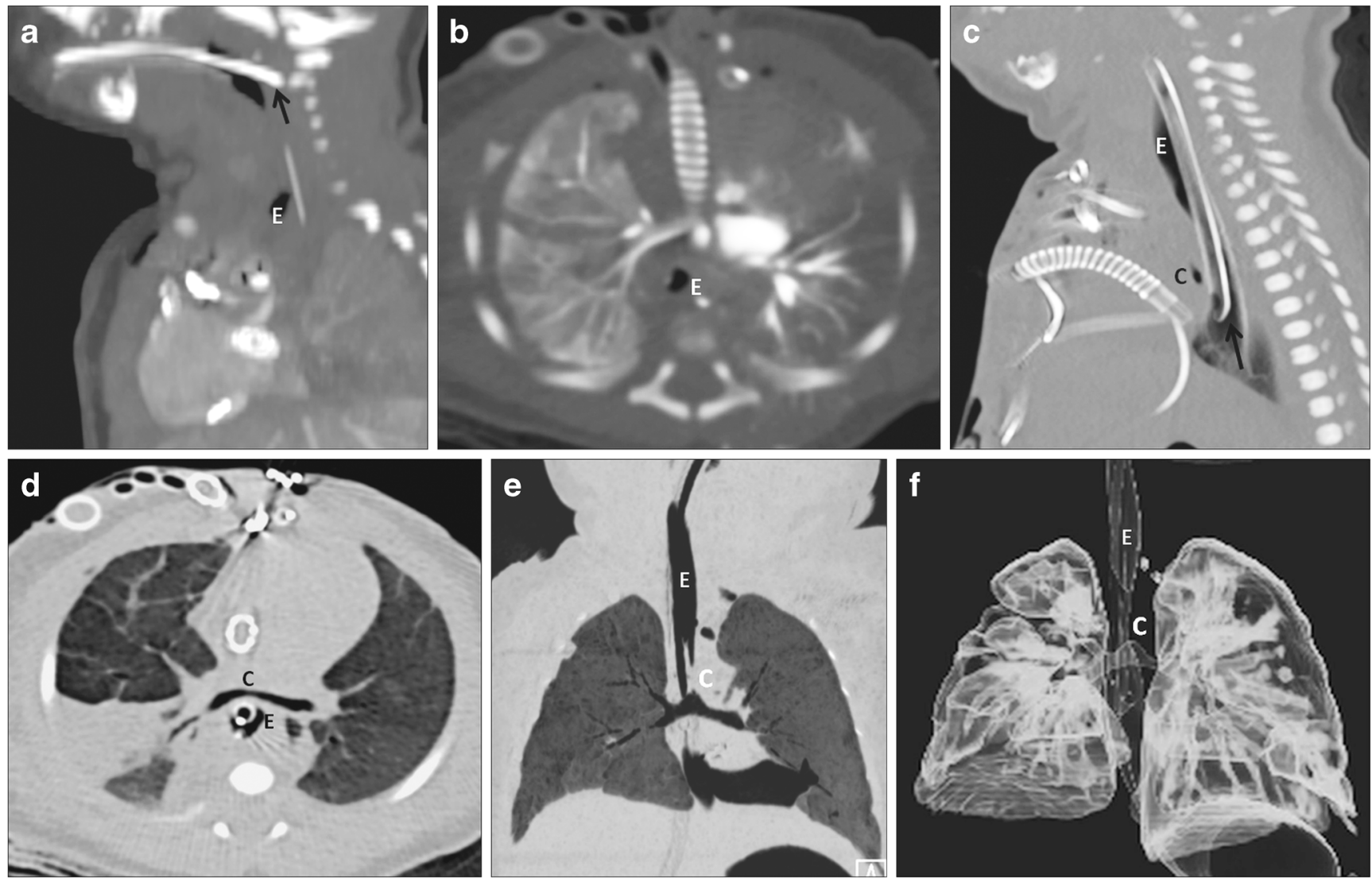

Fig. 5 Optimization of CT diagnosis by airway ventilation in patient 2. a, b Sagittal reconstruction $(a)$ and axial image $(b)$ from the initial CT scan realized after previous ventilation maneuvers by a bag-valve mask attached to an endotracheal tube with the tip (arrow in a) in the esophagus $(E) 5.5 \mathrm{~cm}$ from lips. Carina $(C)$ and main bronchi are not visible and both lungs are completely collapsed. $\mathbf{c}, \mathbf{d}$ Sagittal reformat $(c)$

and axial $(d)$ images of CT scan repeated after advancing the tip of the endotracheal tube and followed by additional ventilation maneuvers, carina $(C)$ and main bronchi are visualized, probably because of the presence of a fistula with the esophagus $(E)$. e, f Minimum-intensity projection $(e)$ and surface-rendered reconstruction $(f)$ better display the anomaly, a type II tracheal agenesis 
examination, even for newborns correctly oxygenated by an extracorporeal membrane oxygenation procedure. During extracorporeal membrane oxygenation, lung ventilation is not mandatory. Nevertheless, during CT examination effective airway opening and lung ventilation is necessary to fully demonstrate the abnormal tracheoesophageal anatomy. Air represents a natural contrast for analyzing the airways on CT, but in newborns with tracheal agenesis airways are poorly aerated. Carina and both mainstem bronchi cannot be spontaneously distended and recognizable, as demonstrated in the second case presented. In our experience, adequate airway ventilation by an endotracheal tube inserted into the esophagus, followed by additional ventilation maneuvers with a bag-valve mask just before CT scan allow for maximizing image quality, improving visualization of the blind-ending trachea and delineating the aberrant tracheoesophageal anatomy. Lung ventilation is an indirect sign of the presence of a tracheoesophageal fistula that is rarely directly recognized [8].

The pressure level needed to open trachea and lungs is linked to the resistance of the tracheoesophageal fistula, and this depends mostly on the diameter but also on the length of this fistula. In most cases this resistance is very high and needed pressures are much higher than those usually used in neonates. An incremental augmentation of ventilation pressure until obtaining good thoracic expansion permits good tracheal and lung aeration.

Secondarily, the use of intravenous contrast material should be considered. It is reported in only three cases in the literature and the benefits and the technical details of injection were not described $[4,5]$. Contrast material obviously allows for better evaluation of the close anatomical relationship with neighboring mediastinal structures, in particular the walls of the esophagus. According to our first experience of diagnosis of this rare disease - examined by an emergent chest CT scan without contrast material and not fully explored with abdominal evaluation - we decided to perform in the second case a complete assessment by a thoraco-abdominal CT. Sonography is the best imaging technique for assessing the abdomen in neonates; however in the second case it was technically difficult to perform because of the presence of extracorporeal membrane oxygenation cannulae passing over the anterior abdominal wall. We believe, however, that contrast material is not essential for the diagnosis of tracheal agenesis and the injection by umbilical catheters is not easy to manage in emergency conditions; when possible an abdominal ultrasound should be performed to search for associated anomalies that are potentially relevant in decision-making. CT findings have a fundamental role in evaluating any possible surgical correction or palliation. Complete diagnosis further allows for adequate counseling between clinicians and parents, and permits a joint decision. Complex, multiple-stage surgical procedures have been described, but at present only type I tracheal agenesis with preservation of a sufficiently long distal trachea opens possibilities for surgical correction. Despite progress in surgical management, to date no tracheal substitute exists that is both non-collapsible and coated with ciliated columnar epithelium. As a consequence, prognosis is very poor [8].

Even if the tracheal agenesis is a very rare congenital anomaly and antenatal diagnosis difficult, radiologists need to be aware of this condition and be prepared for the possibility of encountering it in an emergency setting requiring multidisciplinary management. CT is fundamental for diagnosis of tracheal agenesis and the consequent clinical decision-making. Proper airway ventilation just before CT is essential to evaluating the aberrant tracheoesophageal anatomy. The use of intravenous contrast medium is not compulsory but is advisable for better evaluation of the close mediastinal anatomical relationship.

\section{Compliance with ethical standards}

Conflicts of interest None

\section{References}

1. Mohammed H, West K, Bewick J et al (2016) Tracheal agenesis, a frightening scenario. J Laryngol Otol 130:314-317

2. Floyd J, Campbell DC, Dominy DE (1962) Agenesis of the trachea. Am Rev Respir Dis 86:557-560

3. Billmyre K, Hutson M, Klingensmith J (2015) One shall become two: separation of the esophagus and trachea from the common foregut tube. Dev Dyn 244:277-288

4. De Groot-van der Mooren MD, Haak MC, Lakeman P et al (2012) Tracheal agenesis: approach towards this severe diagnosis. Case report and review of the literature. Eur J Pediatr 171:425-431

5. Strouse PJ, Newman B, Hernandez RJ et al (2006) CT of tracheal agenesis. Pediatr Radiol 36:920-926

6. Hirakawa H, Ueno S, Yokoyama S et al (2002) Tracheal agenesis: a case report. Tokai J Exp Clin Med 27:1-7

7. Coleman AM, Merrow AC, Elluru RG et al (2013) Tracheal agenesis with tracheoesophageal fistulae: fetal MRI diagnosis with confirmation by ultrasound during an ex utero intrapartum therapy (EXIT) delivery and postdelivery MRI. Pediatr Radiol 43:1385-1390

8. Sandu K, Monnier P (2007) Congenital tracheal anomalies. Otolaryngol Clin N Am 40:193-217 